Revue européenne des sciences sociales

European Journal of Social Sciences

XXXVIII-117| 2000

Métaphores et analogies. Schèmes argumentatifs des sciences sociales

\title{
Le cardinal inaccessible. La métaphore comme outil dans la quête de l'infini
}

Henri Volken

\section{(2) OpenEdition}

Journals

Édition électronique

URL : http://journals.openedition.org/ress/717

DOI : $10.4000 /$ ress. 717

ISSN : 1663-4446

Éditeur

Librairie Droz

Édition imprimée

Date de publication : 1 février 2000

Pagination : 131-140

ISBN : 2-600-00409-2

ISSN : 0048-8046

Référence électronique

Henri Volken, «Le cardinal inaccessible. La métaphore comme outil dans la quête de l'infini », Revue européenne des sciences sociales [En ligne], XXXVIII-117| 2000, mis en ligne le 17 décembre 2009, consulté le 19 avril 2019. URL : http://journals.openedition.org/ress/717 ; DOI : 10.4000/ress.717

(c) Librairie Droz 
Henri VOLKEN

\section{LE CARDINAL INACCESSIBLE La métaphore comme outil dans la quête de l'infini}

\begin{abstract}
Je suis tellement pour l'infini actuel, qu'au lieu d'admettre que la nature l'abhorre, comme l'on dit vulgairement, je tiens qu'elle l'affecte partout, pour mieux marquer la perfection de son auteur.
\end{abstract}

Leibniz

Entschulden Sie es gütigst meinem Eifer für die Sache, wenn ich Ihre Güte und Mühe so oft in Anspruch nehme; die Ihnen jüngst von mir zugegangenen Mittheilungen sind für mich selbst so unerwartet, so neu, dass ich gewissermassen nicht eher zu einer gewissen Gemütsruhe kommen kann, als bis ich Ihnen, sehr verehrter Freund, eine Entscheidung über die Richtigkeit derselben erhalten haben werde. Ich kann so lange Sie mir nicht zugestimmt haben, nur sagen: je le vois, mais je ne le crois pas...

Lettre de G. Cantor à R. Dedekind du 29 juin 1877

Les nombres naturels: un, deux, trois..., sont certainement parmi les premiers objets mentaux que l'enfant se construit et utilise de manière systématique. Si l'on rajoute le zéro, placé tardivement ${ }^{1}$ en tête de cette cohorte, on obtient cette structure d'une grande simplicité que tout le monde semble comprendre si facilement: zéro en est le plus petit élément, et tous les autres peuvent être atteints à partir de lui en effectuant un certain nombre de pas unitaires. Tout mystère semble absent de cette construction linéaire. Les pas unitaires correspondent à l'addition du nombre un. Ils permettent la définition de la notion de successeur: chaque nombre possède un successeur. Cela implique qu'il n'y a pas de plus grand nombre, car s'il en existait un, il aurait un successeur et ne serait par conséquent pas le plus grand.

Cette structure représente en quelque sorte un modèle canonique de la notion abstraite de l'ordre linéaire: un élément minimal et une méthode pour construire un successeur immédiat à chaque élément déjà existant. Il n'y a pas de limite dans la construction de nouveaux éléments, pas de surprise non plus. Mais si nous essayons de capter l'ensemble des nombres naturels en un nouveau concept, global et unique, celui-ci devra représenter quelque chose d' «infini», dans le sens de «non terminé », de potentiel. C'est peut-être ici que se situe la première rencontre de l'enfant avec l'idée d'infini.

\footnotetext{
1 Dans la pratique mathématique.
} 
Historiquement, le concept d'infini a été utilisé surtout dans le discours théologique ou philosophique. Dans l'univers grec, il signifiait simplement l'inachevé, l'indéfini et avait parfois une connotation négative ${ }^{2}$. Il suffit de rappeler ici la découverte par les Pythagoriciens de l'irrationalité de certains rapports de grandeurs géométriques. L'existence de ces rapports, qui, dans certains cas, rendaient impossible, car infinie, la recherche d'une mesure commune entre deux grandeurs - le côté d'un carré et sa diagonale par exemple - a été dissimulée le plus longtemps possible par Pythagore et son Ecole ${ }^{3}$. Enfin, Aristote a posé clairement la question de l'existence de l'infini, en distinguant les notions d'infini potentiel et d'infini actuel dans ses études sur le mouvement. Si la notion d'infini potentiel, la possibilité d'une progression non limitée, lui paraissait acceptable, ou du moins inévitable, il rejetait clairement l'existence d'un infini considéré comme une totalité, s'opposant par là d'une certaine façon à Anaximandre et d'autres penseurs grecs.

Quant aux théologiens, leur utilisation de l'infini semble plus positive, même sous la forme de l'infini actuel. Mais ce terme est utilisé principalement pour parler des attributs de Dieu, et en particulier de sa toute-puissance. Les difficultés de ce discours sont nombreuses. L'une d'entre elles réside dans le fait que la toutepuissance divine devrait être infinie, sans toutes fois transgresser les lois de la logique. D'où l'apparition de nombreux paradoxes et énigmes en rapport avec l'utilisation de ce concept.

Or les paradoxes autour de la notion d'infini seront, comme c'est souvent le cas pour les paradoxes, du moins dans le domaine de la logique et des mathématiques, à l'origine du développement de nouvelles théories très fructueuses. Ils étaient déjà courants à l'époque grecque. Les plus célèbres sont probablement les paradoxes inventés par Zénon pour défendre les idées de Parménide, comme le paradoxe d'Achille et de la tortue.

Dans toutes ces discussions, l'infini n'intervient que sous une forme binaire rudimentaire, n'autorisant par conséquent qu'un discours très pauvre: ou une chose est finie, ou elle est infinie. Les mathématiques des $\mathrm{XIX}^{\mathrm{e}}$ et $\mathrm{XX}^{\mathrm{e}}$ siècles ont montré depuis que la situation est beaucoup plus riche ${ }^{4}$. On serait tenté de dire qu'elle est infiniment plus riche. En effet, depuis les travaux de Cantor à partir de 1870 et les débuts de la théorie des nombres transfinis, s'est ouvert un champ fascinant qui donne accès à une vision du monde infini qui le révèle comme stratifié, varié et d'une structure très complexe. L'infini actuel, constitué de l'ensemble des nombres naturels, que certains esprits craignaient de nommer, sous prétexte que peut-être il n'existait pas, n'est que le premier pas, très modeste, dans le monde infini.

L'un des paradoxes les plus spectaculaires, mais aussi des plus féconds, concernant l'infini est celui de la réflexivité. Il s'agit d'un argument centré sur la

Avec des exceptions comme Anaximandre, qui en avait fait un principe universel, mais aussi Empédocle, Archimède et d'une certaine façon Platon.

3 La légende affirme que Hippasos, le disciple de Pythagore qui serait à l'origine de la découverte de l'incommensurabilité de certaines grandeurs, et par là de l'irrationalité de certains nombres, aurait été assassiné.

4 A vrai dire, Leibniz et Newton avaient déjà abordé le sujet par le biais des «infiniment petits » au XVII ${ }^{\mathrm{e}}$ siècle. 
notion de «taille» d'un ensemble. On admettra volontiers que deux ensembles sont de même taille si l'on peut mettre les éléments de l'un en correspondance biunivoque avec les éléments de l'autre: à chaque élément du premier correspond exactement un élément du second. Dans le monde fini cela ne pose aucun problème, et traduit fidèlement notre intuition de l'égalité de la taille de deux ensembles. Les choses semblent se gâter lorsqu'on veut franchir le pas de l'infini en conservant cette même méthode de comparaison.

En effet, à chaque nombre pris dans l'ensemble des nombres naturels, on peut faire correspondre son double. A l'inverse, à chaque nombre pair, on peut faire correspondre sa moitié. Ainsi on crée une correspondance bi-univoque entre les nombres naturels et les nombres pairs : il y aurait autant de nombres pairs qu'il y a de nombres! Que sont devenus les nombres impairs? Ce paradoxe a été longtemps utilisé comme argument pour « démontrer» que l'infini ne pouvait pas exister, du moins sous la forme d'un infini objet, d'un infini actuel. Comment un ensemble pourrait-il avoir «la même taille» que l'une de ses parties propres?

La réflexivité est donc cette propriété d'un ensemble infini qui permet l'existence d'une correspondance bi-univoque avec l'un de ses sous-ensembles. C'est précisément cette propriété qui a été le plus souvent utilisée comme argument pour infirmer l'existence de l'infini actuel. Or le mathématicien allemand Dedekind a retourné la situation de manière lumineuse et proposé la réflexivité comme définition des ensembles infinis: un ensemble est infini si l'une de ses parties peut être mise en correspondance bi-univoque avec le tout. Le principe communément admis que le tout «est plus grand» que ses parties ne s'applique plus, dans cette perspective, que dans un monde fini!

C'est le point de départ d'une véritable conquête de l'infini par les mathématiques et le premier exemple d'un langage et d'une théorie qui permettent de voir dans l'infini un paysage subtilement diversifié plutôt qu'un amas informe, inachevé. Nous allons retracer quelques étapes de cette conquête en montrant comment la métaphore, qui consiste à attribuer à un objet mathématique précis le terme polysémique d' «infini», se transforme progressivement, et joue un rôle de guide dans cette exploration.

La découverte du transfini est essentiellement l'œuvre d'un seul homme: Georg Cantor ${ }^{5}$, génial mathématicien allemand de la deuxième moitié du siècle passé. Ses travaux sur les fondements des nombres réels l'ont amené progressivement à s'intéresser de manière plus approfondie à la notion d'infini et plus particulièrement à l'opposition infini actuel/infini potentiel qui remonte à Aristote. Et pour avoir pris au sérieux et avoir défendu fermement la notion d'infini actuel, pour l'avoir fait passer du stade de métaphore à celui de concept mathématique, Cantor a soulevé l'hostilité d'une bonne partie de ses contemporains, avant que ses idées ne s'imposent à la majorité des mathématiciens de noter siècle.

Nous allons retracer quelques étapes importantes de cette quête de l'infini en utilisant un formalisme le plus restreint possible. Néanmoins, les objets mathématiques n'étant pas accessibles en dehors de leur représentation, il nous a fallu choisir une notation suffisamment précise pour rendre visible un peu de la magie de ce fascinant paysage découvert par Cantor.

1845-1918, fondateur de la théorie des ensembles et des nombres transfinis. 
Reprenons l'exemple si simple de la suite des nombres naturels $0,1,2, \ldots$ qui nous fournit une image constructive de l'infini, mais une image banale et quotidienne: quel que soit le nombre considéré, n'importe qui peut lui trouver un successeur, c'est-à-dire un nombre encore plus grand. Jusqu'où peut-on aller? Et au bout, y a-t-il des nombres «infinis »? La réponse est indirecte: on peut les construire, ils ne provoquent pas de contradiction. Le problème épineux de l'existence d'objets mathématiques est ainsi, comme si souvent, transformé par le mathématicien en un autre problème: y a-t-il une théorie cohérente de ces objets?

Pour répondre à cette dernière question, il est indispensable de préciser la représentation que nous avons des nombres et de situer celle-ci dans le langage des ensembles afin de pouvoir profiter de son potentiel constructif. Nous allons choisir la définition suivante:

«Un nombre est l'ensemble de tous les nombres plus petits que lui ».

Comment une telle définition, qui peut paraître absurde, peut-elle être utilisée? Prenons l'exemple du 0, qui, étant le plus petit nombre, est donc représenté par l'ensemble vide, noté habituellement $\emptyset$, puisque précisément il n'y a pas de nombres plus petits. Le nombre 1, par contre, contient le 0 . Il est donc représenté par l'ensemble $\{0\}$. Et ainsi de suite. Nous obtenons ainsi la représentation des nombres suivante:

$$
0=\varnothing, 1=\{0\}, 2=\{0,1\}, 3=\{0,1,2\}, \ldots
$$

L'avantage de cette représentation est de plusieurs ordres. D'une part chaque nombre, considéré comme ensemble, contient autant d'éléments qu'il le «dit». Il s'agit donc bien d'une représentation naturelle et explicite. D'autre part, le langage des ensembles est directement applicable avec toutes ses possibilités constructives. Finalement, la relation «est plus petit que» est représentée de manière interne par la relation d'appartenance d'un élément à un ensemble. Un exemple: dans cette représentation des nombres, « 1 est plus petit que 3 » signifie exactement la même chose que «1 appartient à 3 ». Cela montre que la relation d'ordre entre nombres naturels possède un modèle intérieur très simple dans notre représentation ensembliste: il s'agit de la relation d'appartenance. Donc dans cette perspective, un nombre contient effectivement tous les nombres plus petits que lui.

Pour l'instant, nous sommes toujours dans le domaine des nombres naturels que tout le monde connaît et utilise quotidiennement. Et parler de nombre «infini» n'est rien d'autre qu'une métaphore pour un nombre «extrêmement grand», un nombre qui dépasse notre imagination arithmétique habituelle, et qui soit plus grand que tous les nombres imaginables. Or justement, dans notre définition des nombres, rien ne nous empêche d'envisager le concept d'ensemble de tous les nombres naturels! Cet ensemble est parfaitement défini et ne contient que des objets innocents. Il est tentant de le considérer comme candidat au statut de premier nombre infini et de transformer ainsi la métaphore en réalité. En effet, il contient tous les nombres plus petits que lui, tous les nombres naturels, et rien d'autre. Il s'agit donc bien d'un «nombre», même s'il est d'une nature un peu différente. La terminologie mathématique lui attribue habituellement le nom de $\omega$.

Nous avons donc une image, un nom et surtout un concept mathématique précis qui nous permet de faire un premier pas dans ce paysage inconnu, au-delà du 
fini. La différence essentielle qui distingue $\omega$ des autres nombres réside notamment dans le fait qu'il n'est le successeur direct d'aucun nombre, autrement dit qu'il ne possède pas de prédécesseur immédiat. Par contre, il possède un successeur.

La construction qui associe à chaque nombre son «successeur», correspond à l'opération d'addition d'une unité. Cette opération est définie en toute généralité de la manière suivante: Si $\alpha$ est un ensemble, alors $S(\alpha)=\alpha \cup\{\alpha\}$, c'est-à-dire l'ensemble qui regroupe $\alpha$ et tous les éléments qu'il contient. Dans le monde des nombres, cela constitue précisément la construction du successeur immédiat d'un nombre, conformément à la représentation proposée.

Par exemple: $\mathrm{S}(3)=3 \cup\{3\}=4=3+1$. De chaque nombre naturel on peut ainsi passer au suivant en appliquant cette opération. A partir de 0 , on peut atteindre - ou construire - de cette manière tous les nombres naturels, mais pas $\omega$, le premier des nombres «infinis ». L'itération de la simple opération d'addition ne nous permet pas de sortir du fini. Par contre, notre définition des nombres nous a fourni une procédure pour aller au-delà: il suffit de former l'ensemble de tous les nombres déjà construits et de l'interpréter comme le plus petit nombre plus grand que tous les autres.

En résumé, nous avons la situation suivante: en partant du nombre 0, on peut construire tous ses successeurs, $1,2,3,4, \ldots$ et obtenir ainsi tous les nombres naturels. En regroupant tous ces nombres en un ensemble, celui-ci devient a son tour un nombre, que nous avons appelé $\omega$. Rien ne nous empêche alors de construire la suite de ses successeurs, qu'on appellera $\omega+1, \omega+2, \omega+3, \ldots$ et ainsi de suite. Tous ces nombres sont infinis mais représentent des formes d'infini différentes. Cantor a introduit le terme de nombres ordinaux pour caractériser ces nombres généralisés. Les deux principes de construction, l'idée de successeur et l'idée du regroupement de tous les nombres déjà construits en un nouveau nombre, permettent d'explorer l'infini de manière systématique. Un nombre obtenu par le premier procédé est un ordinal-successeur, un nombre obtenu par le second, un ordinallimite. $\omega$ est donc le premier ordinal limite. La suite des ordinaux continue ainsi :..., $\omega+4, \omega+5, \omega+6, \ldots$ qui sont des successeurs de $\omega$. Tous les ordinaux construits jusque-là sont ensuite regroupés, en application du deuxième principe en un nombre $\omega+\omega$, appelé aussi $\omega .2$, le deuxième ordinal-limite. L'infini se révèle, à la lumière de cette construction, d'une richesse incroyable, et $\omega$, pourtant souvent la limite de l'imagination dans ce domaine, n'est qu'un petit pas - le premier - au delà du fini. Ce paysage nouveau apparaît clairement dans sa stratification: $\omega .2, \omega .2+1, \omega .2+2, \ldots \omega .3, \ldots, \omega .4, \ldots, \omega . \omega\left(\right.$ ou $\left.\omega^{2}\right), \ldots, \omega^{3}, \ldots, \omega^{\omega}, \ldots$ avec des structures de plus en plus subtiles.

Le simple fait de prendre la métaphore de l'«infini » au sérieux, de lui faire correspondre un objet mental, $\omega$, construit à partir de concepts élémentaires, a suffi pour développer un langage et une théorie qui permettent d'appréhender un univers d'une grande complexité, et surtout de «mesurer la longueur» de certaines catégories infinies et ainsi de pouvoir les comparer de manière beaucoup plus précise.

Le mathématicien D. Hilbert ${ }^{6}$ a inventé une illustration amusante pour montrer toute la richesse de l'univers des nombres ordinaux, mais surtout pour souligner

1862-1943. 
les différences entre le monde fini et le monde infini. Supposons un hôtel immense, dit Hilbert, mais avec un nombre fini de chambres. S'il est plein, il ne pourra plus accueillir d'hôtes nouveaux, malgré son grand nombre de chambres. Prenons ensuite un hôtel avec un nombre infini de chambres, disons $\omega$ chambres, qui lui aussi est entièrement occupé. S'il vient un nouvel hôte, poursuit Hilbert, nous pouvons toujours lui trouver une chambre, il suffit de déplacer les hôtes résidents de la manière suivante: chaque hôte passe dans la chambre suivante selon la numérotation des chambres, c'est-à-dire dans la chambre portant le numéro $n+1$ si sa chambre portait le numéro n. Il suffit ensuite de placer le nouvel arrivant dans la première chambre. On voit aisément comment caser, en appliquant les mêmes idées, 10,100, 1000 ou n'importe quel nombre fini de personnes supplémentaires dans cet hôtel, qui pourtant était plein avant leur venue! Avec un peu de réflexion, on voit qu'on peut caser même un nombre plus grand de personnes: $\omega$, ou $\omega+1$, ..., et même $\omega^{\omega}$, et plus. Peut-on alors caser n'importe quel nombre (ordinal!) de personnes? La réponse est non.

Il y a des nombres ordinaux trop « grands » pour cela. Revenons à la définition des ordinaux qui est à l'origine de notre construction. Nous avons considéré l'ensemble des nombres naturels ordonnés par la relation d'appartenance. L'image naïve correspondante est celle d'une chaîne. Il y a une idée sous-jacente d'ordre. Donc si nous mesurons quelque chose à l'aide des nombres ordinaux, c'est la «longueur» de cette chaîne. Et c'est effectivement l'un des usages des nombres dans la vie courante: nous dénombrons des objets en les parcourant et en égrenant parallèlement la suite un, deux, trois etc. Ce que nous avons obtenu de plus, en construisant les ordinaux, c'est un moyen de continuer cette action de dénombrer dans le domaine infini. Nous pouvons parler de manière cohérente d'une file de longueur $\omega$, pour une chaîne semblable aux nombres naturels, ou d'une file de longueur $\omega+1$, pour une telle chaîne mais avec un dernier élément, et ainsi de suite.

Une deuxième utilisation habituelle des nombres est celle de mesurer la «taille» d'un ensemble d'objets. Il n'y a cette fois pas de considération d'ordre, il ne s'agit pas de caractériser les propriétés d'un enchaînement, mais bien plutôt d'avoir un moyen de comparer la «taille», la «masse» ou la «grandeur» d'un ensemble, tous ces termes résultant de points de vue différents sur la question, selon les contextes de leur emploi.

Les nombres, vus sous cet aspect, sont définis à l'aide d'un concept très différent de celui d'ordre. C'est la réponse à la question: «quand deux ensembles ontils la même taille?» qui fournit la clé pour une définition rigoureuse. On peut certainement considérer, comme nous l'avons déjà évoqué, que deux ensembles possèdent la même taille, si à chaque élément du premier on peut faire correspondre de manière bi-univoque un élément du second. Dans ce cas, on dira que ces deux ensembles ont la même cardinalité. La cardinalité d'un nombre naturel est ce nombre lui-même, qui par là, devient un nombre cardinal, ou plus simplement, un cardinal. Et pour tout ensemble fini, son cardinal est simplement le nombre naturel qui a la même cardinalité que lui, c'est-à-dire le nombre qui, considéré comme ensemble, peut être mis en correspondance bi-univoque avec lui. Dans le monde fini, il n'y a pas de différence entre cardinal et ordinal. Le nombre 0 et tous ses successeurs sont à la fois des ordinaux et des cardinaux, dissimulant d'une certaine façon les deux rôles différents qu'ils jouent. Dès que nous 
avons mis un pied dans l'infini - il suffit pour cela de prendre au sérieux la construction de $\omega$ - la situation devient plus subtile.

La fiction de l'hôtel imaginaire de D. Hilbert nous a montré que les ordinaux $\omega$ et $\omega+1$ ont la même taille. Il y a une correspondance univoque dans les deux sens entre les personnes déjà présentes avant l'arrivée d'un nouvel hôte, et les personnes logées finalement à l'hôtel. Cette correspondance est réalisée très simplement par les numéros des chambres: le numéro 17, par exemple, relie le premier occupant de cette chambre, à l'occupant final. Donc les ordinaux $\omega$ et $\omega+1$ sont différents mais correspondent au même cardinal, puisqu'ils mesurent des ensembles de même taille. Pour les cardinaux, Cantor a introduit la terminologie suivante: pour le premier cardinal infini, il utilise le symbole $\aleph_{0}$. Bien sûr, le premier ordinal et le premier cardinal infinis sont identiques : $\boldsymbol{\aleph}_{0}=\omega$.

Certains ordinaux sont donc aussi des cardinaux, mais ce n'est pas toujours le cas, comme nous venons de le voir. Un cardinal peut être défini comme un ordinal dont la cardinalité est supérieure à celle de tous les ordinaux plus petits que lui. C'est le cas pour $\omega$ mais pas pour $\omega+1$ et ses successeurs. Tous les ensembles de cardinal $\boldsymbol{\aleph}_{0}$ sont appelés dénombrables. Ce qui signifie qu'on peut placer leurs éléments dans le même ordre que les nombres naturels, autrement dit, qu'on peut numéroter leurs éléments dans le sens courant du terme. Le prochain cardinal, $\boldsymbol{\aleph}_{1}$ n'apparaîtra que très loin dans la hiérarchie des ordinaux, montrant que l'univers infini, mais dénombrable, possède une structure très compliquée.

Et comme il ne peut pas y avoir de plus grand ordinal - son successeur serait encore plus grand - il n'y a pas non plus de plus grand cardinal. Il ne peut pas non plus exister l'ensemble de tous les ordinaux, puisqu'il serait lui-même un ordinal. Cantor a montré, dans un théorème célèbre, que l'ensemble des parties d'un ensemble possède un cardinal supérieur à celui de l'ensemble de départ. Cela signifie que la construction mentale qui consiste à regrouper en un nouvel ensemble toutes les classes ou catégories d'un ensemble d'éléments, crée un objet d'une complexité essentiellement supérieure. Par conséquent, pour chaque cardinal donné, il existe un cardinal plus grand.

En particulier, le cardinal de l'ensemble des parties de $\omega$ est plus grand que $\aleph_{0}$, il est donc non dénombrable. Si l'on applique ces idées de mesure de l'infini à d'autres systèmes de nombres que nous utilisons, les nombres rationnels et les nombres réels, nous arrivons à des constatations surprenantes. Les nombres rationnels, c'est-à-dire les nombres que l'on obtient comme quotients de deux nombres naturels, sont dénombrables. On peut écrire la liste des nombres rationnels. Par contre il y a un nombre non dénombrable de nombres réels, comme Cantor l'a démontré par sa méthode de la diagonalisation. Supposons pour simplifier que les réels situés dans l'intervalle [0,1] puissent être numérotés et présentés en une liste exhaustive sous la forme décimale habituelle. On peut facilement construire un réel dont le développement décimal diffère du premier de la liste par la première décimale, du deuxième par la deuxième décimale et ainsi de suite. (D'où le terme de diagonalisation.) Pour toute énumération des nombres réels, on peut ainsi trouver un nombre réel qui ne se trouve pas dans la liste.

Il y a donc essentiellement plus de réels que de naturels. L'introduction des cardinaux permet de préciser l'une des questions importantes en rapport avec l'infini: où se situe le continu dans la hiérarchie des cardinaux? Le continu étant le plus souvent assimilé par les mathématiciens à la structure des nombres réels, la 
question revient à savoir si le cardinal des réels est $\aleph_{1}$ ou un cardinal supérieur, puisqu'il ne peut pas s'agir de $\boldsymbol{\aleph}_{0}$ comme nous venons de voir. La question posée est donc:

$\operatorname{card}(\Re)=\aleph_{1}$, ou si l'on veut, $\operatorname{card}(\mathrm{P} \omega)=\aleph_{1}$, puisque $\operatorname{card}(\Re)=\operatorname{card}(\mathrm{P} \omega)^{7}$.

A cette question, Cantor n'a pas pu répondre. Il l'a formulée sous forme d'hypothèse du continu: $\operatorname{card}\left(\mathrm{P} \aleph_{0}\right)=\operatorname{card}\left(\aleph_{1}\right)$.

Cette question cruciale sur l'infini n'a été résolue qu'au $\mathrm{XX}^{\mathrm{e}}$ siècle, en deux étapes différentes. En 1936, Gödel a montré qu'il était impossible de démontrer que l'hypothèse était fausse et, en 1963, Cohen a complété le résultat en montrant qu'il était impossible de démontrer ${ }^{8}$ que l'hypothèse était vraie. Il s'agit donc d'un énoncé indépendant, ce qui signifie qu'il est légitime d'imaginer des infinis dans lesquels ce résultat est vrai, et d'autres dans lesquels il est faux ${ }^{9}$.

Jusqu'ici, le fait d'avoir accepté l'idée que $\omega$ existe, et qu'il réalise la métaphore de l'infini nous a permis de construire deux systèmes de nombres capables de mesurer des ensembles infinis. Mesurer dans les deux sens différents de «mesurer la longueur» et de «mesurer la taille». Nous allons brièvement esquisser l'utilisation de ces notions dans la construction, ambitieuse, d'un univers des ensembles. Il s'agit de la réponse à la question: de quel objet parle le mathématicien lorsqu'il parle d'un «ensemble», dans quel univers le situe-t-il?

Il s'agit d'une construction basée sur les ordinaux qui fonctionnent comme un fil conducteur dans la complexification progressive de cet univers. Il s'agit à nouveau d'un univers stratifié et les différentes couches sont construites de la manière suivante:

la première strate est formée de l'ensemble vide. Ensuite, si la strate portant le numéro $\alpha$ est déjà construite, la suivante contiendra toutes ses parties. Cela pour tout ordinal-successeur. Pour un ordinal-limite, comme $\omega$ par exemple, la strate correspondante sera formée de la réunion de toutes les strates déjà créées. En termes un peu plus formels, voici la procédure de construction de l'univers des ensembles:

$$
\begin{array}{ll}
\mathrm{V}(0)=\emptyset . & \\
\mathrm{V}(\alpha+1)=\mathrm{P}(\mathrm{V}(\alpha)) & \text { pour tout ordinal-successeur } \alpha . \\
\mathrm{V}(\lambda)=\cup\{\mathrm{V}(\alpha) \mid \alpha<\lambda\} & \text { pour tout ordinal limite } \lambda .
\end{array}
$$

On peut démontrer que tout ensemble se situe quelque part dans l'une de ces strates, dans l'un de ces univers partiels, $\mathrm{V}(\alpha)$. C'est dans cet univers construit au fil des ordinaux, que se déroule la théorie des ensembles des mathématiciens, telle qu'elle a été développée par Cantor et à sa suite par Zermelo, Fraenkel, Bernays, Gödel, von Neumann et bien d'autres. Les différentes théories axiomatiques qui ont été développées dans ce cadre sont probablement consistantes, c'est-à-dire exemptes de contradictions, mais cela n'est pas encore démontré. Par contre il

P $\omega$ désigne l'ensemble des parties de $\omega$.

8 Démontrer dans le cadre d'une théorie axiomatique des ensembles comme Zermelo-Fraenkel ou Bernays-Gödel.

9 Il s'agit du même statut que celui que possède le cinquième postulat d'Euclide. 
existe un lien intéressant entre la consistance de la théorie des ensembles et les cardinaux très grands.

La particularité sous-jacente à la définition des ordinaux, qui finalement nous a permis un accès à l'«infini », se trouve dans le fait que certains ordinaux sont atteignables par le bas, en remontant l'échelle des successeurs en quelque sorte, alors que d'autres, les ordinaux-limite, ne le sont que par des opérations mentales plus complexes, comme la formation d'ensembles. En ce qui concerne les cardinaux, nous avons vu qu'ils paraissent plus difficiles d'accès. Après le premier cardinal infini, $\boldsymbol{\aleph}_{0}$, il semble que le chemin soit très long jusqu'au suivant, $\boldsymbol{\aleph}_{1}$. Toutes les opérations de successeurs et de réunion d'ensembles ne nous font pas sortir du monde dénombrable. Et il faut l'opération P, celle qui permet de construire l'ensemble des parties, pour atteindre le premier cardinal non dénombrable. Ensuite il faudra imaginer des opérations de plus en plus sophistiquées pour dépasser les divers paliers de la cardinalité. Et là, nous entrons dans un domaine assez étrange des mathématiques: la théorie des grands cardinaux.

Il n'est, bien sûr, plus possible de donner dans ce court exposé les définitions explicites concernant les différentes catégories de grands cardinaux. Le but de cette taxinomie est d'abord de classifier l'atteignabilité des cardinaux en fonction des opérations utilisées pour les construire. Ainsi un cardinal est appelé régulier si toute réunion d'un de ses sous-ensembles de cardinalité plus petite que lui, reste de cardinalité plus petite que lui. Et un cardinal régulier est inaccessible s'il n'est pas un cardinal-successeur ${ }^{10}$. Le terme est approprié puisqu'un tel nombre est très difficile à construire. Ensuite les cardinaux peuvent se révéler hyper-inaccessibles, voire hyper-hyper-inaccessibles. Plus compliqués encore, les cardinaux de Mahlo. Ou finalement les cardinaux indescriptibles, les ineffables, les cardinaux de Ramsey, et les cardinaux extensibles. Et tout au bout, un objet déjà entrevu et redouté par Cantor, l'infini absolu $\Omega$, qui lui ne peut pas être un ordinal. Cantor a utilisé le terme de «système inconsistant» pour le caractériser, montrant par là sa perplexité. Nous allons discrètement le passer sous silence.

Parmi les définitions de classes de grands cardinaux, il en est une qui joue un rôle particulier en ce qui concerne la non-contradiction de la théorie des ensembles. Il s'agit des cardinaux fortement inaccessibles. Un tel cardinal est non-dénombrable, régulier, et le cardinal de l'ensemble des parties de tout ensemble de cardinalité plus petite, est lui-même plus petit que le cardinal. Cela signifie qu'il n'est pas constructible à partir de cardinaux plus petits en utilisant les opérations ensemblistes ordinaires, y compris l'opération $\mathrm{P}$ dont nous avons parlé.

$\mathrm{Vu}$ la difficulté de construire un tel cardinal, une question se pose. Y a-t-il un cardinal inaccessible? La question se révèle capitale, car un théorème important montre que s'il existe un tel cardinal, appelons-le $\kappa$, alors l'univers partiel V( $\kappa)$ est un modèle de la théorie axiomatique des ensembles. Ce qui signifie que l'existence d'un cardinal fortement inaccessible démontre la non-contradiction de cette théorie. La question de l'existence ou de la construction d'un tel cardinal est donc de la même difficulté que celle de la consistance de la théorie des ensembles.

10 C'est-à-dire s'il ne correspond pas à un ordinal-successeur. 
C'est un résultat remarquable, qui éclaire d'un jour nouveau la portée de ce petit «tour de passe-passe ${ }^{11}$ qui consiste à recueillir tous les nombres $0,1,2, \ldots$ en un ensemble et de le réinterpréter à son tour comme nombre. Et finalement, la structure complexe obtenue, la vision toute neuve qu'elle permet sur l'infini, ou plutôt sur les infinis, vaut bien cet effort d'imagination. A moins qu'il s'agisse d'un acte de foi. Mais c'est un fait que l'«infini», en passant de la simple métaphore pour «ce qui n'est pas limité » à une réalité mathématique fascinante, n'a rien perdu de son charme. Ni de son mystère.

Institut de mathématiques appliquées

Université de Lausanne

11 Pour lequel il pourrait passer, en présence d'un méfiance forte envers cette « désinvolture ontologique». 